\title{
A novel plasma lncRNA ENST00000416361 is upregulated in coronary artery disease and is related to inflammation and lipid metabolism
}

\author{
PING LI $^{1 *}$, XINXIN YAN ${ }^{1 *}$, GUIDONG XU ${ }^{2}$, ZHI PANG ${ }^{1}$, JIAYI WENG ${ }^{2}$, JUAN YIN $^{1}$, \\ MEIFEN LI ${ }^{1}$, LAN YU ${ }^{1}$, QIAN CHEN ${ }^{1}$ and KANGYUN SUN ${ }^{2}$ \\ ${ }^{1}$ Institute of Digestive Diseases and Nutrition and ${ }^{2}$ Department of Cardiology, \\ The Affiliated Suzhou Hospital of Nanjing Medical University, Suzhou, Jiangsu 215008, P.R. China
}

Received August 21, 2019; Accepted March 10, 2020

DOI: $10.3892 / \mathrm{mmr} .2020 .11067$

\begin{abstract}
Coronary artery disease (CAD) is a serious threat to human health and a major cause of mortality worldwide. Long noncoding RNAs (lncRNAs) affect the occurrence and development of CAD via the regulation of cell proliferation and apoptosis, inflammatory responses and lipid metabolism. Screening methods and therapeutic strategies for CAD have been extensively studied. The present study analyzed clinical indexes of 187 patients with CAD and 150 healthy subjects. The data showed significant differences in diabetes mellitus, hypertension, high-density lipoprotein level and smoking history between the CAD group and the control group. A series of differentially expressed lncRNAs were detected in the plasma samples of three patients with CAD by high-throughput sequencing. Reverse transcription-quantitative (RT-q)PCR data revealed that the expression level of the novel lncRNA ENST00000416361 was 2.3-fold higher in the plasma of 50 patients with CAD compared with the 50 control subjects. Receiver operating characteristic (ROC) curves were generated, and the area under the ROC curve was 0.7902 . Knockdown of ENST00000416361 in human umbilical vein endothelial cells markedly downregulated interleukin- 6 and tumor necrosis factor- $\alpha$ levels. In addition, sterol regulatory element binding transcription factor (SREBP)1 and SREBP2 were upregulated
\end{abstract}

Correspondence to: Dr Kangyun Sun, Department of Cardiology, The Affiliated Suzhou Hospital of Nanjing Medical University, 242 Guangji Road, Suzhou, Jiangsu 215008, P.R. China

E-mail: skywj66@163.com

Dr Qian Chen, Institute of Digestive Diseases and Nutrition, The Affiliated Suzhou Hospital of Nanjing Medical University, 242 Guangji Road, Suzhou, Jiangsu 215008, P.R. China

E-mail: sdfeycq@sina.com

*Contributed equally

Key words: coronary artery disease, long noncoding RNA, ENST00000416361, inflammation, lipid metabolism, biomarker in patients with $\mathrm{CAD}$, and they were positively correlated with the expression of ENST00000416361. RT-qPCR further demonstrated that knockdown of ENST00000416361 led to the downregulation of SREBP1 and SREBP2. Overall, the novel lncRNA ENST00000416361 may be associated with CAD-induced inflammation and lipid metabolism, and it may serve as a potential biomarker for CAD.

\section{Introduction}

Coronary artery disease (CAD) is a type of heart disease caused by atherosclerotic lesions of coronary arteries, which is characterized by stenosis or obstruction of the vascular cavity that further leads to myocardial ischemia, hypoxia or necrosis (1). Atherosclerosis, the primary cause of cardiovascular disease, is a chronic inflammatory process involving inflammatory plaques, different types of cells with multiple complex interactions and abnormal lipoprotein deposits in the arterial wall $(2,3)$. Hypertension, diabetes mellitus, tobacco consumption, alcohol consumption, sex (male), obesity, dyslipidemia, genetic variance and a lack of physical activity are commonly known risk factors for CAD $(4,5)$.

Long noncoding RNAs (lncRNAs) are a class of noncoding RNAs with a transcript length $>200$ bp that lack protein-coding potential. Due to their cell and tissue specificity, lncRNAs in blood, plasma or urine can be used as biomarkers and therapeutic targets for cancer and numerous other diseases $(6,7)$. An increasing number of studies have shown that lncRNAs are abnormally expressed in a variety of diseases and have important roles in cell proliferation, apoptosis and invasion (8-10).

It has been demonstrated that IncRNAs are closely associated with the occurrence and development of CAD, including CAD-induced pathological changes such as vascular smooth muscle cell proliferation, apoptosis, lipid metabolism and inflammation $(11,12)$. Various IncRNAs have been considered promising regulatory genes or biomarkers of $\mathrm{CAD}$, such as lncPPAR $\delta$, H19 imprinted maternally expressed transcript, maternally expressed 3, antisense noncoding RNA in the INK4 locus (ANRIL), mitochondrially encoded long non-coding cardiac associated RNA, lincRNA-p21, CoroMarker, NONHSAT112178, Novlnc6, metastasis associated lung 
adenocarcinoma transcript 1, myocardial infarction associated transcript and steroid receptor RNA activator (SRA) $(13,14)$. In atherosclerosis, lincRNA-p21 has been identified to inhibit proliferation and induce apoptosis in vascular smooth muscle cells (15). In addition, the G-A-A-G haplotype of lincRNA-p21 is correlated with a decreased risk of CAD (16).

CoroMarker (lncRNA AC100865.1), abhydrolase domain containing 16A, and IL21R antisense RNA 1 were identified by microarray and confirmed by reverse transcription-quantitative (RT-q) PCR in peripheral blood mononuclear cells (PBMCs) (17). It has been shown that in CAD the CoroMarker is significantly upregulated and has high specificity and sensitivity (17). Studies have also demonstrated that CoroMarker can positively regulate foam cell formation, arteriosclerosis, inflammation and the associated immune responses, and can inhibit cell apoptosis $(17,18)$.

A number of lncRNAs have been shown to be associated with CAD, however key lncRNAs in patients with CAD from different regions and different ethnicities are likely to differ, so further studies are required. In the present study, high-throughput sequencing was adopted to identify differentially expressed lncRNAs and identify a biomarker for CAD according to the local resident (Suzhou, Jiangsu, China). Further study of the mechanism of the lncRNA was performed to identify new targets for the early diagnosis and treatment of clinical CAD. These results also provided a theoretical basis for the further study of the molecular mechanism of CAD.

\section{Materials and methods}

Clinical characteristics of patients. A total of 187 patients with CAD, aged 42-85 years, were enrolled in the Department of Cardiology of Nanjing Medical University Affiliated Suzhou Hospital in China between October 2016 and August 2018. The diagnosis of CAD was based on angiographic evidence of at least one segment of a major coronary artery, including the left anterior descending, left circumflex and right coronary arteries, with $\geq 50 \%$ stenosis (19). Exclusion criteria were as follows: i) Patients with severe primary diseases, such as liver, kidney and hematopoietic system; ii) patients with acute infection, trauma or other operations; iii) patients with myocardial infarction, cerebral infarction, cancer and other major diseases; iv) patients with alcoholism and drug addictions; v) patients $>85$ years; and vi) pregnant patients. During the same period, 150 healthy subjects were selected from patients attending check-up appointments. The routine blood test results (WBC: $4-10 \times 10^{9} / \mathrm{L}$, HGB: $120-170 \mathrm{~g} / 1$ (male), 110-150 g/l (female), PLT: 100-300x10 $/ 1$ ), blood pressure (60-90/90-140 mmHg), blood glucose (4-6.1 mmol/l), blood lipid (total cholesterol: $2.8-5.17 \mathrm{mmol} / 1$, triglycerides: $0.56-1.7 \mathrm{mmol} / \mathrm{l}$, cholesterol lipids: $2.8-5.17 \mathrm{mmol} / \mathrm{l}$ ) and electrocardiogram (ECG; physician judgment) of those 150 healthy subjects were within the normal ranges. The study was approved by the Ethics Committee of Nanjing Medical University. Informed consent was obtained from the patients and their families.

Collection and preparation of blood samples. Peripheral blood samples (4-6 ml) were collected from patients with CAD and healthy subjects using EDTA vacuum anticoagulant blood vessels. After incubation for $30 \mathrm{~min}$ at $4^{\circ} \mathrm{C}$, the blood samples were centrifuged at $200 \mathrm{x}$ g for $10 \mathrm{~min}\left(4^{\circ} \mathrm{C}\right)$. Then, the plasma was recentrifuged at $1,600 \mathrm{x}$ g for $10 \mathrm{~min}\left(4^{\circ} \mathrm{C}\right)$ to remove debris. PBMCs were separated from the blood cells with Ficoll-Paque PLUS density gradient media (GE Healthcare) as previously described $(20,21)$.

lncRNA-Seq high-throughput sequencing. Plasma samples from 3 patients with CAD and 3 healthy subjects were subjected to IncRNA-Seq high-throughput sequencing, followed by bioinformatics analysis performed by CloudSeq Biotech. Total RNA $(1 \mu \mathrm{g})$ was used to remove the ribosomal (r)RNAs using Ribo-Zero rRNA Removal kits (Illumina, Inc.) following the manufacturer's protocols.

Fragments per kilobase million (FPKM) of the expression profiles of IncRNAs and mRNAs was calculated using Cuffdiff software (v2.2.1, part of Cufflinks) (22). Fold change and P-value were calculated based on FPKM to identify the differentially expressed lncRNAs and mRNAs. Gene Ontology (GO) pathway enrichment analysis was performed based on the differentially expressed mRNAs $(23,24)$.

Competing endogenous (ce)RNA analysis of lncRNAs. An RNA transcript with micro (mi)RNA response elements (MREs) may serve as a competing endogenous (ce)RNA. ceRNAs include pseudogene transcripts, lncRNAs, circular RNAs and mRNAs, and these transcripts can compete for the same MREs to exert their biological functions. Potential target miRNAs were predicted with in-house miRNA target prediction software, based on TargetScan and miRanda software (25-31). Cytoscape software (v3.7.0) was used to visualize the ceRNA networks with the target miRNAs (32).

To further analyze the metabolic pathway of lncRNA ENST00000416361, a ceRNA network map was constructed according to the corresponding target miRNAs and mRNAs. The physiological effect of lncRNA ENST00000416361 was studied in depth by analyzing the biological functions of related miRNAs and mRNAs.

Cell culture. HUVECs (human umbilical vein endothelial cells) were purchased from CoWin Biosciences. HUVECs were cultured in DMEM (Thermo Fisher Scientific, Inc.) supplemented with $100 \mathrm{U} / \mathrm{ml}$ streptomycin, $100 \mathrm{U} / \mathrm{ml}$ penicillin and $10 \%$ (v/v) FBS (Gibco; Thermo Fisher Scientific, Inc.) under $37^{\circ} \mathrm{C}$ and $5 \%$ (v/v) $\mathrm{CO}_{2}$ conditions.

Design of small interfering (si)RNAs and cell transfection. The siRNAs were designed and synthesized by Suzhou GenePharma Co., Ltd. The sequences of si-ENST00000416361 were: Sense (5'-3'), CCCAACAGCUCAUUGAGAATT and antisense (5'-3'), UUCUCAAUGAGCUGUUGGGTT. The sequences of siRNA-NC were: Sense (5'-3'), UUCUCCGAACG UGUCACGUTT and antisense (5'-3'), ACGUGACACGUUCGG AGAATT. A total of $100 \mathrm{nM}$ siRNA was used for each transfection. A negative control with fluorescein was used to identify cell transfection efficiency. Cell transfection was performed using Lipofectamine $^{\mathrm{TM}} 3000$ (Invitrogen; Thermo Fisher Scientific, Inc.) following the manufacturer's protocol. Knockdown efficiency and related gene expression were measured after $12 \mathrm{~h}$.

RNA isolation and RT-qPCR. Plasma RNA was extracted by TRIzol ${ }^{\mathrm{TM}}$ LS Reagent (Invitrogen; Thermo Fisher 
Scientific, Inc.), and $1 \mu \mathrm{g}$ RNA was transcribed into cDNA by $4 \mu 15 \mathrm{X}$ PrimeScript ${ }^{\mathrm{TM}}$ RT Master Mix (RR036A) (Takara Bio, Inc.), up to $20 \mu \mathrm{l}$ with RNase free $\mathrm{dH}_{2} \mathrm{O}$. RNAiso Plus (Takara Bio, Inc.) was used to extract RNA from PBMCs and HUVECs, followed by reverse transcription to cDNA with PrimeScript $^{\mathrm{TM}}$ RT Master Mix (RR036A) (Takara Bio, Inc.). RNA quality was analyzed with an ultra-micro nucleic acid analyzer (Hangzhou Allsheng Instruments Co., Ltd.).

RT-qPCR was performed using SYBR ${ }^{\circledR}$ Premix Ex Taq $^{\mathrm{TM}}$ II (Takara Bio, Inc.) to detect relative expression using the standard protocols on the LightCycler ${ }^{\circledR} 480$ Instrument II Real-Time PCR Detection system (Roche Diagnostics). $\beta$-actin was used as the internal reference. PCR reaction conditions were: $95^{\circ} \mathrm{C}$ for $30 \mathrm{sec} ; 40$ cycles of $95^{\circ} \mathrm{C}$ for $5 \mathrm{sec}$ and $60^{\circ} \mathrm{C}$ for $20 \mathrm{sec} ; 95^{\circ} \mathrm{C}$ for $0 \mathrm{sec}, 65^{\circ} \mathrm{C}$ for $15 \mathrm{sec}$ and $95^{\circ} \mathrm{C}$ for $0 \mathrm{sec}$. The percentage of agarose gel was $2 \%$. The primers for lncRNAs, the lipid-associated genes SREBP1, FAS, ACC1, DGAT2, SREBP2, ApoE, ApoA-I, Cpt1a (33) and $\beta$-actin used in the RT-qPCR were synthesized by Genewiz, Inc. The primer sequences are listed in Table I. All measurements were performed in triplicate. Relative expression data were analyzed with the formula $2^{-\Delta \Delta \mathrm{Cq}}$ (34). GraphPad Prism 7 (GraphPad Software, Inc.) was used to analyze the differences in expression.

Cytokine detection. The human Th1/Th2 subgroup test kit (CellGene Biotech Co., Ltd.) was used to detect the levels of the inflammatory cytokines interleukin (IL)-2, IL-4, IL-6, IL-10, interferon- $\gamma$ and tumor necrosis factor- $\alpha$ (TNF- $\alpha$ ). The standard solution and standard curves were prepared according to the manufacturer's protocols. The mixed microsphere solution was centrifuged at $200 \mathrm{x}$ g for $5 \mathrm{~min}\left(4^{\circ} \mathrm{C}\right)$. Subsequently, the same volume of microsphere buffer solution was added and incubated for $30 \mathrm{~min}$ in the dark at room temperature. For each tube, $25 \mu 1$ microsphere mixture, $25 \mu 1$ sample and $25 \mu$ l fluorescent detection reagent was added, and the solution was incubated for $2.5 \mathrm{~h}$ at room temperature. After washing with $1 \mathrm{ml}$ of PBS, the mixture was centrifuged at $200 \times \mathrm{g}$ for $5 \mathrm{~min}$ at room temperature, and the precipitate was diluted in $100 \mu 1$ of PBS solution. Finally, cytokine detection was performed using BD FACSCalibur ${ }^{\mathrm{TM}}$ (Becton-Dickinson and Company).

Statistical analysis. GraphPad Prism 7 software was used for statistical analysis. All data were obtained from at least 3 independent experiments and are expressed as the mean \pm SD. One-way ANOVA followed by Dunnett's test was performed to analyze multiple group comparisons of quantitative data and Student's t-test was performed for analyzing two-group comparisons of quantitative data. A Chi-square test was used for category data. Correlation was confirmed by Pearson's correlation analysis. $\mathrm{P}<0.05$ was considered statistically significant.

\section{Results}

Pathological differences between patients with CAD and healthy subjects. A total of 187 patients with CAD (diagnosed with at least one vascular $\geq 50 \%$ stenosis) and 150 healthy subjects were selected for the experiment. The clinical
Table I. The primer sequences.

\begin{tabular}{ll}
\hline Gene & \multicolumn{1}{c}{ Primer sequence } \\
\hline ENST00000416361 & 5'-CTCATTGAGAACGGGCCATG-3' \\
& 5'-CATGTTTCAGAGAGCACCGG-3' \\
SREBP1 & 5'-GGAGGGGTAGGGCCAACGGCCT-3' \\
SREBP2 & 5'-CATGTCTTCGAAAGTGCAATCC-3' \\
& 5'-CAAGATGCACAAGTCTGGCG-3' \\
FAS & 5'-GCTTCAGCACCATGTTCTCCTG-3' \\
& 5'-ACAGGGACAACCTGGAGTTCT-3' \\
ACC1 & 5'-CTGTGGTCCCACTTGATGAGT-3' \\
& 5'-GTTGCACAAAAGGATTTCAG-3' \\
DGAT2 & 5'-CGCATTACCATGCTCCGCAC-3' \\
& 5'-CGGTCCCCAATCACCTCATC-3' \\
ApoE & 5'-GGGATGTTCCAGTTCTGCCA-3' \\
& 5'-CCCAGGTCACCCAGGAACT-3' \\
ApoA-I & 5'-TTCCGATTTGTAGGCCTTCAA-3' \\
& 5'-ATCGAGTGAAGGACCTGGC-3' \\
Cpt1a & 5'-AGCTTGCTGAAGGTGGAGGT-3' \\
& 5'-TGCTTTACAGGCGCAAACTG-3' \\
& 5'-TGGAATCGTGGATCCCAAA-3' \\
& 5'-CACGAAACTACCTTCAACTCC-3' \\
& 5'-CATACTCCTGCTTGCTGATC-3'
\end{tabular}

characteristics of the enrolled patients and healthy controls are presented in Table II. The data showed that $82.9 \%$ of patients with CAD had hypertension, $28.9 \%$ had diabetes, $45 \%$ had a history of smoking and were male, all of which were significantly increased compared with those in the control group. In addition, high-density lipoprotein (HDL) levels were significantly decreased in patients with CAD relative to the controls.

Differential expression profiles of IncRNAs. Plasma samples from 3 patients with CAD and 3 healthy controls were examined via high-throughput sequencing methods. The 3 patients with CAD were screened based on their clinical records, in order to exclude the interference of other diseases as well as to avoid including the effects of smoking and drinking. In the 3 healthy controls, all the physical examination indexes were in the normal range, and the interference of other diseases was excluded. All the physical examination indexes were in the normal range, and the interference of other diseases was excluded of the normal group. By analyzing FPKM, fold changes and P-values using the Cuffdiff software, 66 upregulated and 86 downregulated lncRNAs were identified. Cluster analysis, scatter diagram and volcano plots show the differentially expressed lncRNAs with a $\mathrm{P} \leq 0.05$ and fold change $\geq 2.0$ (Fig. 1).

GO pathway analysis of differentially expressed lncRNAs. Target genes of lncRNAs were predicted based on their proximity to the lncRNAs, and GO pathway analysis of biological processes was performed based on the identified target genes. The bar plot shows the top fold enrichment value of 
Table II. Clinical characteristics of patients with CAD and controls.

\begin{tabular}{|c|c|c|c|c|c|}
\hline \multirow[b]{2}{*}{ Characteristics } & \multicolumn{2}{|c|}{ Sequencing samples } & \multicolumn{3}{|c|}{ All clinical samples collected } \\
\hline & CAD $(n=3)$ & $\mathrm{CON}(\mathrm{n}=3)$ & $\mathrm{CAD}(\mathrm{n}=187)$ & $\mathrm{CON}(\mathrm{n}=150)$ & P-value \\
\hline Age, years & 60.33 & 57.78 & $67.5 \pm 17.5$ & $62.06 \pm 18.06$ & $>0.050$ \\
\hline Sex, male $(\%)$ & $2(66.7)$ & $2(66.7)$ & $115(61.5)$ & $78(52)$ & 0.083 \\
\hline $\operatorname{BMI}\left(\mathrm{kg} / \mathrm{m}^{2}\right)$ & 24.32 & 23.99 & 25.13 & 25.68 & $>0.050$ \\
\hline Diabetes mellitus (\%) & $0(0)$ & $0(0)$ & $54(28.9)$ & $22(14.7)$ & 0.002 \\
\hline Hypertension (\%) & $1(33.4)$ & $0(0)$ & $155(82.9)$ & $58(38.7)$ & $<0.001$ \\
\hline $\mathrm{TC}(\mathrm{mmol} / \mathrm{l})$ & 3.56 & 3.69 & 4.14 & 4.589 & 0.066 \\
\hline $\mathrm{TG}(\mathrm{mmol} / \mathrm{l})$ & 1.57 & 1.77 & 1.7 & 1.56 & 0.679 \\
\hline $\operatorname{HDL}(\mathrm{mmol} / \mathrm{l})$ & 0.98 & 1.08 & 1.02 & 1.19 & 0.002 \\
\hline $\operatorname{LDL}(\mathrm{mmol} / \mathrm{l})$ & 2.13 & 2.21 & 2.56 & 2.89 & 0.171 \\
\hline VLDL (mmol/l) & 0.34 & 0.5 & 0.56 & 0.53 & 0.425 \\
\hline $\operatorname{ApoA}(g / 1)$ & 1.32 & 1.11 & 1.21 & 1.25 & 0.776 \\
\hline ApoB (g/l) & 0.71 & 0.69 & 0.82 & 0.83 & 0.421 \\
\hline Lipoprotein A & 200.67 & 162.09 & 207.24 & 177 & 0.051 \\
\hline $\mathrm{CHE}$ & 8.41 & 8.74 & 7.8 & 8.04 & 0.773 \\
\hline TBA & 5.23 & 5.15 & 5.77 & 5.56 & 0.894 \\
\hline Smoking (\%) & $0(0)$ & $0(0)$ & $84(45)$ & $40(26.7)$ & 0.001 \\
\hline Drinking (\%) & $0(0)$ & $0(0)$ & $37(19.8)$ & $20(13.3)$ & 0.116 \\
\hline
\end{tabular}

$\mathrm{P}<0.05$ was regarded as significant. Chi-square test and unpaired t-test were used. CAD, coronary artery disease; CON, control; BMI, body mass index; n, number of patients; TC, total cholesterol; TG, triglyceride; HDL, high-density lipoprotein; LDL, low-density lipoprotein; VLDL, very low-density lipoprotein; ApoA, apolipoprotein A1; ApoB, apolipoprotein B; CHE, cholinesterase; TBA, total bile acid.
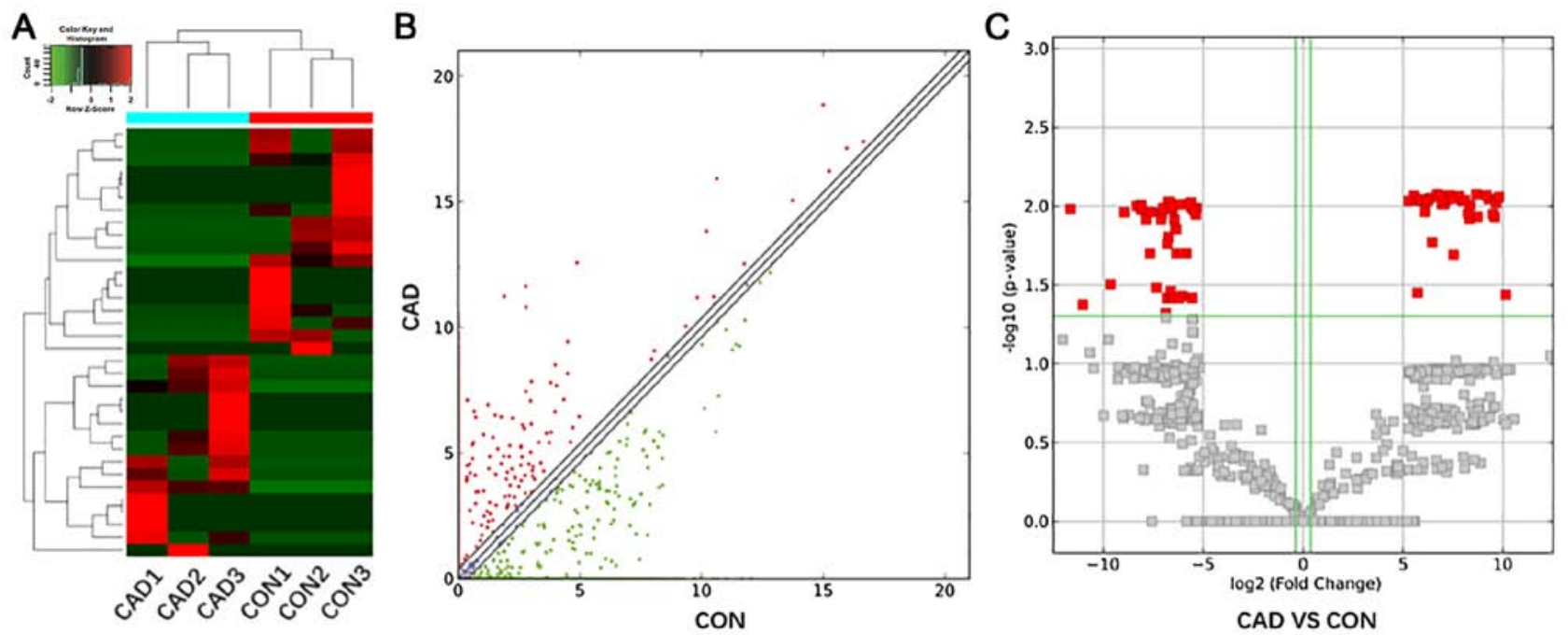

Figure 1. Differentially expressed lncRNAs in CAD and control groups identified by high-throughput sequencing. (A) Cluster analysis of differentially expressed lncRNAs in the CAD (CAD1, CAD2 and CAD3) and control groups (CON1, CON2 and CON3). Upregulated genes are represented as red and downregulated ones are represented as green. (B) Scatter diagram of two groups was generated according to the expression values. Red indicates upregulation and green indicates downregulation. The default fold change threshold was 2.0. (C) Volcano plots were generated using fold-change and P-value data, and the red rectangles represent the expression differences of lncRNAs. IncRNA, long noncoding RNA; CAD, coronary artery disease; CON, control.

the significantly enriched terms (Fig. 2). The target genes of upregulated lncRNAs were associated with serotonin metabolic process, primary amino compound metabolic process and nuclear localization signal (NLS)-bearing protein import into nucleus (Fig. 2A and C). The target genes of the downregulated IncRNAs were mainly involved in primitive streak formation, semicircular canal development, embryonic viscerocranium morphogenesis, regulation of RNA export from nucleus and facial nerve morphogenesis (Fig. 2B and D).

Target lncRNA selection. The top 5 upregulated and downregulated lncRNAs with $\mathrm{P}<0.01$ were selected, and overlapping sequences were excluded (Table III). 
A

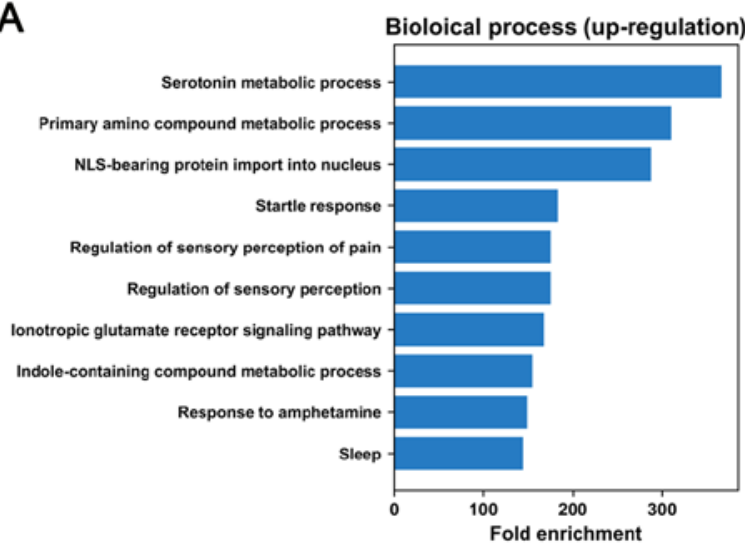

C

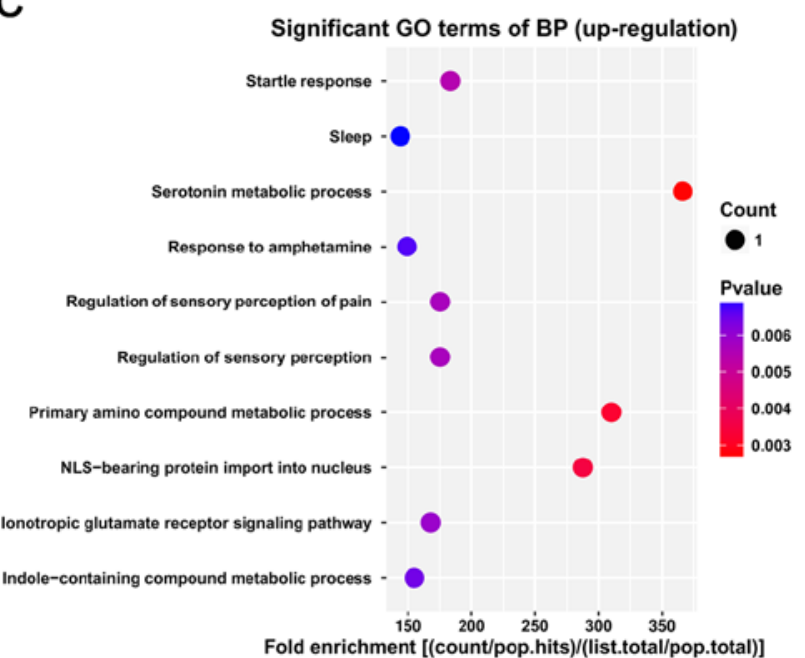

B

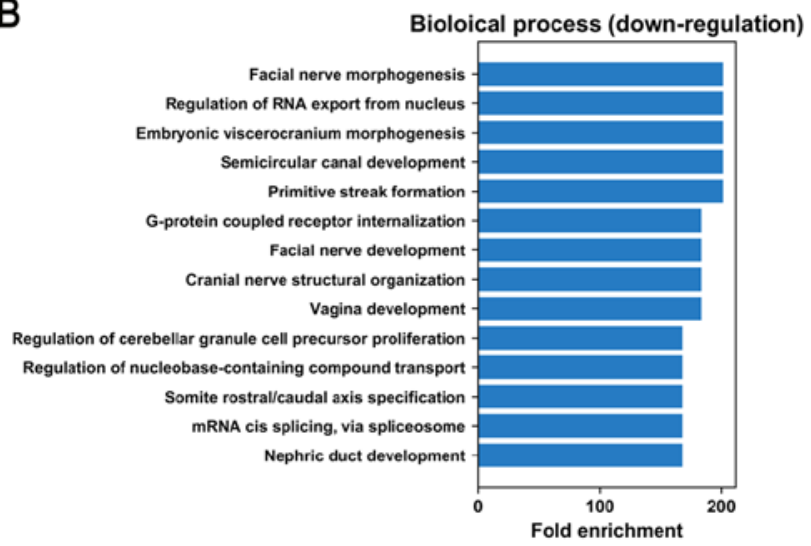

D

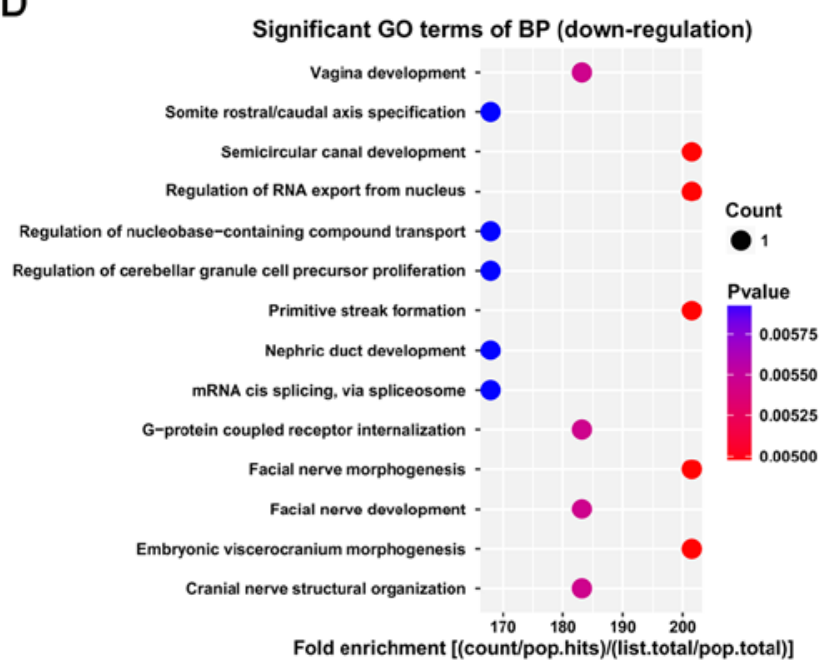

Figure 2. GO pathway analysis of target genes. (A and C) GO pathway analysis of upregulated lncRNAs. (B and D) GO pathway analysis of downregulated lncRNAs. GO, Gene Ontology; lncRNA, long noncoding RNA.
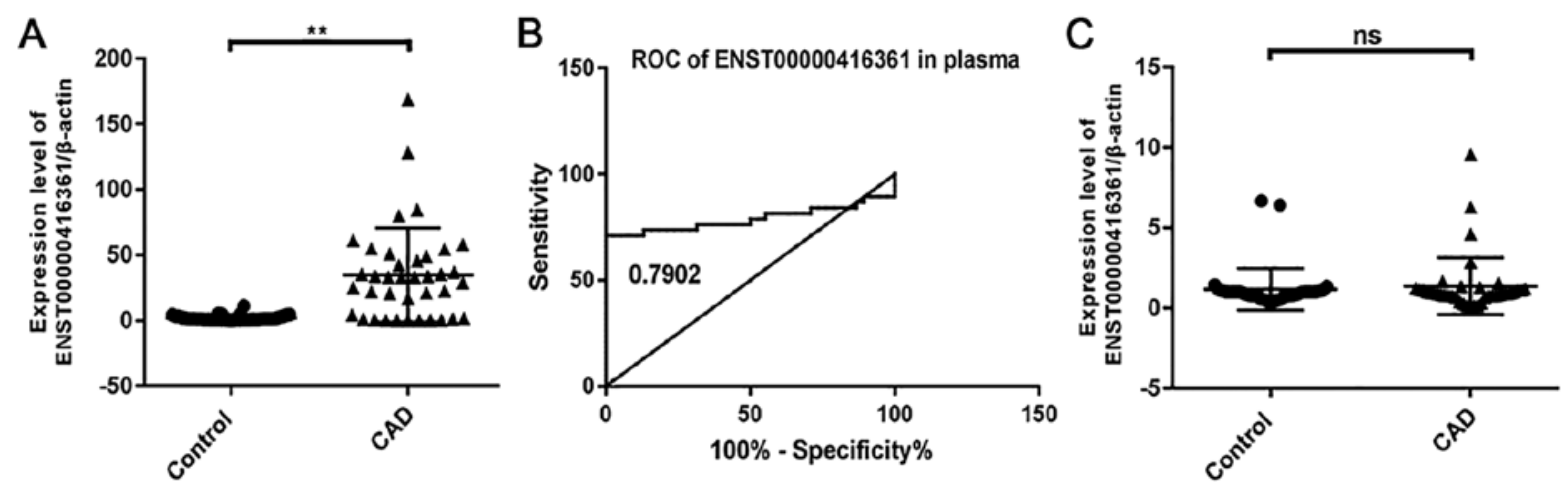

Figure 3. Expression level and ROC curve analysis of lncRNA ENST00000416361. (A) Expression level of ENST00000416361 in peripheral blood plasma samples $(\mathrm{n}=50) .{ }^{* *} \mathrm{P}<0.01$. (B) ROC curve of ENST00000416361 expression in peripheral blood plasma samples. (C) Expression level of ENST00000416361 in peripheral blood mononuclear cells $(\mathrm{n}=50)$. ROC, receiver operating characteristic; lncRNA, long noncoding RNA; CAD, coronary artery disease; n.s., no significant difference.

IncRNA ENST00000416361 is significantly upregulated in the plasma of patients with CAD. A total of 3 pairs of primers were designed for each IncRNA, and the results showed that ENST00000416361 was upregulated in the peripheral blood plasma samples of patients with CAD (Fig. 3A). However, the upregulation trend of ENST00000416361 was not obvious in PBMCs (Fig. 3C). The relative expression levels of these lncRNAs in plasma and PBMC samples from 50 patients with
CAD and 50 healthy controls were verified by RT-qPCR. Then, 50 samples per group were randomly selected from the 187 patients with CAD and 150 controls enrolled in the study. The results indicated that the expression level of ENST00000416361 in patients with CAD was 2.3-fold higher compared with that in the normal group, and $\mathrm{P}<0.01$ was used as a cut-off value to indicate a statistically significant difference. A ROC curve was generated for analyzing the early-stage 


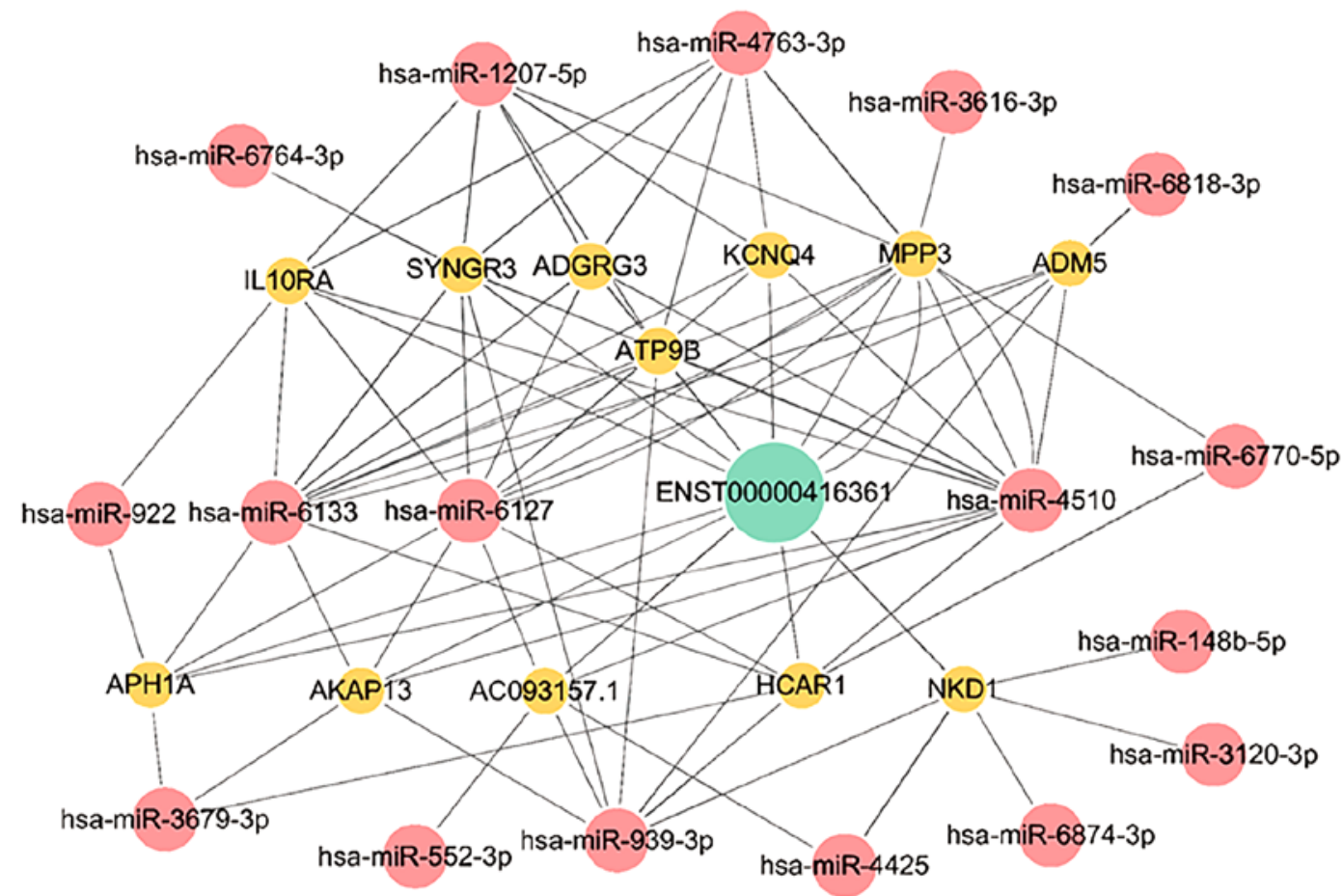

Figure 4. CeRNA network map of lncRNA ENST00000416361-associated miRNAs and mRNAs. The ceRNA network map was constructed using Cytoscape software. The green circle is lncRNA, the red circles are miRNAs and the yellow circles are mRNAs. ceRNA, competing endogenous RNA; lncRNA, long noncoding RNA; miRNA/miR, microRNA.
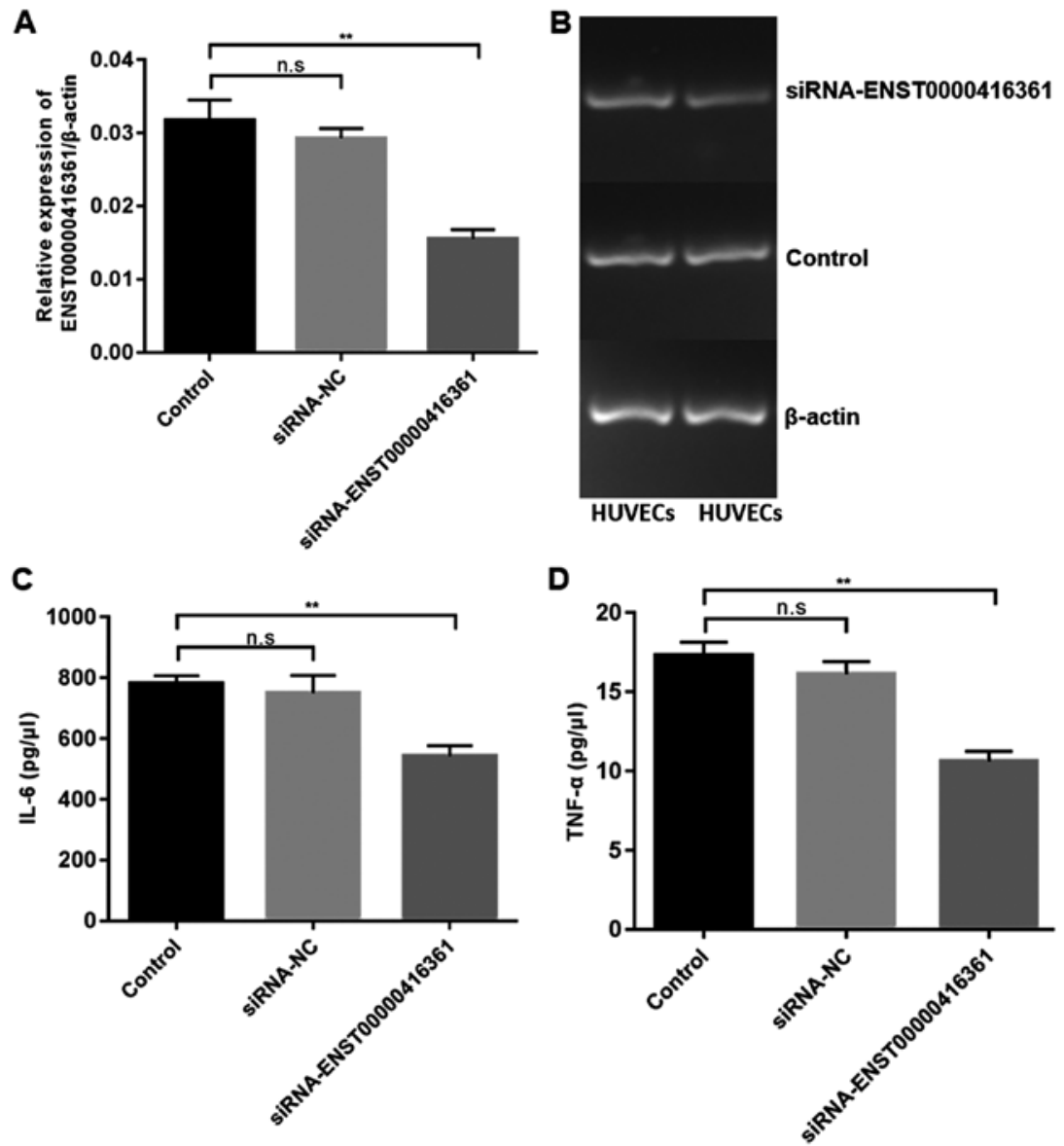

Figure 5. Transfection of si-ENST00000416361 downregulated inflammatory factors. (A and B) Transfection efficacy of si-ENST00000416361 in HUVECs determined by reverse transcription-quantitative PCR. $\beta$-actin was used as the internal reference. (C and D) Inflammatory cytokines IL-6 and TNF- $\alpha$ in the cell supernatant were measured by the human Th1/Th2 subgroup test kit. ${ }^{* *} \mathrm{P}<0.01$. HUVECs, human umbilical vein endothelial cells; IL, interleukin; TNF- $\alpha$, tumor necrosis factor- $\alpha$; siRNA, small interfering RNA; n.s., no significant difference; NC, negative control. 

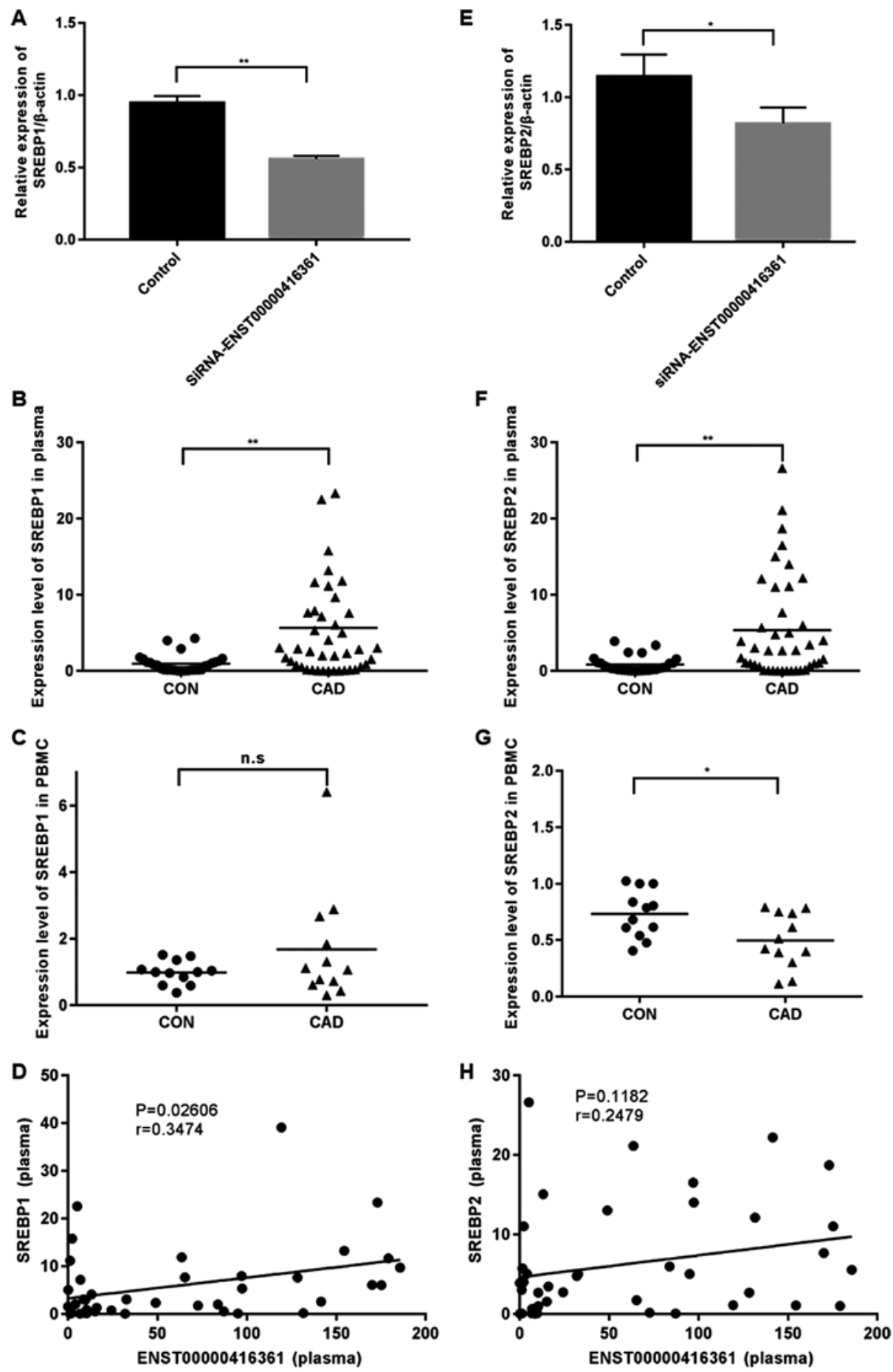

Figure 6. Expression levels of SREBP1 and SREBP2 in HUVECs and plasma samples. (A and E) Relative expression levels of (A) SREBP1 and (E) SREBP2 were detected by RT-qPCR following ENST00000416361 knockdown in HUVECs. (B and F) Plasma levels of (B) SREBP1 and (F) SREBP2 in patients with $\mathrm{CAD}$ and control subjects determined by RT-qPCR (CAD $=42$ cases, $C O N=28$ cases). ${ }^{* * *} \mathrm{P}<0.01$. (C and G) Relative expression of (C) SREBP1 and $(\mathrm{G}) \mathrm{SREBP} 2$ in peripheral blood mononuclear cells determined by RT-qPCR (CAD=12 cases, $\mathrm{CON}=12$ cases). $\mathrm{P}<0.05$. (D and $\mathrm{H}$ ) Pearson's correlation scatter plots revealed the correlation between plasma levels of (D) ENST00000416361 and (H) SREBP1/SREBP2 analyzed with GraphPad Prism 7.0 (n=41). SREBP, sterol regulatory element binding transcription factor; HUVECs, human umbilical vein endothelial cells; RT-qPCR, reverse transcription-quantitative PCR; $\mathrm{CAD}$, coronary artery disease; $\mathrm{CON}$, control; n.s, no significant difference.

screening and diagnostic potential of ENST00000416361 in CAD (Fig. 3B). The area under the curve was 0.7902, indicating that the plasma level of ENST00000416361 may represent a useful clinical diagnostic biomarker for CAD.

IncRNA ENST00000416361 serves as a ceRNA. Bioinformatics analysis was conducted to explore the gene metabolic pathway of lncRNA ENST00000416361. A ceRNA network map was constructed with the relevant miRNAs and mRNAs (Fig. 4).

Among these related mRNAs, synaptogyrin 3, membrane palmitoylated protein 3 , adhesion $\mathrm{G}$ protein-coupled receptor G3, aph-1 homolog A, $\gamma$-secretase subunit, hydroxycarboxylic acid receptor 1, ATPase phospholipid transporting 9B and potassium voltage-gated channel 
Table III. Target lncRNA selection.

\begin{tabular}{lcccclcl}
\hline Transcript_ID & Fold change & P-value & FDR & Regulation & LncRNA_source & LncRNA_length (bp) & Relationship \\
\hline ENST00000416361 & inf & 0.009 & 0.400733 & Up & Ensembl & 2,102 & Intergenic \\
ENST00000565257 & inf & 0.010 & 0.400733 & Up & Ensembl & 1,406 & Intergenic \\
ENST00000568324 & inf & 0.010 & 0.400733 & Up & Ensembl & 3,102 & Intergenic \\
uc003pxg.1 & inf & 0.009 & 0.400733 & Up & UCSC knowngene & 2,183 & Nidirectional \\
ENST00000607001 & inf & 0.010 & 0.400733 & Up & Ensembl & 744 & Nidirectional \\
ENST00000418006 & -inf & 0.009 & 0.400733 & Down & Ensembl & 2,350 & Intergenic \\
ENST00000564481 & -inf & 0.010 & 0.400733 & Down & Ensembl & 992 & Nidirectional \\
ENST00000537921 & -inf & 0.011 & 0.400733 & Down & Ensembl & 477 & Nidirectional \\
ENST00000554522 & -inf & 0.009 & 0.400733 & Down & Ensembl & 1,701 & Intergenic \\
ENST00000425077 & -inf & 0.010 & 0.400733 & Down & Ensembl & 422 & Intergenic \\
\hline
\end{tabular}

$\mathrm{P}<0.05$ was considered to indicate significance. FDR, false discovery rate; lncRNA, long noncoding RNA.

subfamily Q member 4 were associated with membrane structures.

The miRNA-939 expression is downregulated in patients with CAD. miRNA-939 inhibits angiogenesis by targeting human-catenin proteins, thus destroying the integrity of blood vessels. Overexpression of miRNA-939 in HUVECs can significantly inhibit cell proliferation, adhesion and angiogenesis but increase cell migration (35).

Inflammatory factors IL- 6 and TNF- $\alpha$ are significantly downregulated following lncRNA ENST00000416361 knockdown. Transfection of si-ENST00000416361 in HUVECs exhibited a 50\% knockdown efficiency (Fig. 5A and B). Furthermore, transfection of si-ENST00000416361 led to the downregulation of IL-6 (Fig. 5C) and TNF- $\alpha$ (Fig. 5D).

Lipid-associated genes SREBP1 and SREBP2 are positively correlated with the expression of ENST00000416361.It was identified that SREBP1 and SREBP2 were significantly upregulated in plasma samples of patients with CAD (Fig. 6B and F), but not in PBMCs (Fig. 6C and G). Knockdown of ENST00000416361 led to the significant downregulation of SREBP1 and SREBP2 (Fig. 6A and E). Pearson's correlation scatter plots were generated based on the plasma levels of ENST00000416361 and SREBP, and it was indicated that ENST00000416361 could affect the occurrence and development of CAD by interacting with SREBP. The levels of SREBP1 $(\mathrm{P}=0.02606, \mathrm{r}=0.3474)$ and SREBP2 $(\mathrm{P}=0.1182, \mathrm{r}=0.2479)$ were both positively correlated with the expression of ENST00000416361, which was more pronounced in the former gene (Fig. 6D and H).

\section{Discussion}

lncRNAs have been identified to be closely associated with the occurrence and development of CAD (36). These differentially expressed lncRNAs have attracted increasing attention due to their value in disease diagnosis and prognosis. In the present study, high-throughput sequencing and pathway analysis were conducted to find the differentially expressed lncRNAs in the peripheral blood of patients with CAD. The top upregulated lncRNAs were primarily associated with serotonin metabolic process, primary amino compound metabolic process and NLS-bearing protein import into nucleus. By analyzing clinical indexes of patients with CAD and healthy subjects, it was identified that HDL levels were significantly decreased in patients with CAD. The expression pattern of lncRNA ENST00000416361 was determined in plasma samples and PBMCs, indicating that the plasma level of IncRNA ENST00000416361 may be a potential biomarker for clinical CAD screening.

Knockdown of ENST00000416361 significantly decreased the levels of the inflammatory factors IL- 6 and TNF- $\alpha$. A growing number of studies have shown that IL- 6 and TNF- $\alpha$ are closely related to CAD progression. It has been demonstrated that the serum levels of IL- 6 and TNF- $\alpha$ are increased in patients with CAD and are positively correlated with miR-342-5p expression (37). Decreased serum levels of C1q/TNF- $\alpha$ related protein 12 (CTRP12) have been identified in patients with CAD compared to controls, and IL-6 and TNF- $\alpha$ are negatively correlated with CTRP12 in patients with CAD (38). It has been suggested that inflammatory cytokines, particularly IL-6, may be elicited in response to an acute inflammatory stressor, thus contributing to the progression of CAD $(39,40)$. The $\mathrm{C}$ allele of rs1800795 is located on the IL-6 gene promoter, and IL-6 rs1800795-tobacco smoking and IL-6 rs1800795-alcohol consumption interactions have been associated with increased CAD risk (41). IncRNA ANRIL affects the progression of CAD by targeting miR-181b, activating the $\mathrm{NF}-\kappa \mathrm{B}$ signal pathway and inducing the release of inflammatory factors such as IL-6 and TNF- $\alpha$ (42).

In addition, SREBP1 and SREBP2, which were significantly upregulated in CAD plasma samples, were downregulated following the knockdown of lncRNA ENST00000416361. SREBPs are important molecules in lipid synthesis and are encoded by 2 SREBP genes, SREBP1 and SREBP2 (43). SREBP1 is primarily distributed in the liver and adrenal gland and it is closely associated with genes involved in fatty acid metabolism (44). SREBP2 is widely expressed and participates in cholesterol biosynthesis and metabolism $(44,45)$. Increasing studies have focused on the correlation between SREBP and CAD. SREBP is upregulated in patients with CAD and is a cardiovascular risk factor for the severity of CAD and poor lipid control (46). Another study identified that the recessive allele 
(TT vs. CT+CC) of SREBP1 rs9902941 is highly expressed in patients with CAD compared with the normal control (47). miRNA-33a and miRNA-33b are intron miRNAs encoded by the intron regions of SREBP1 and SREBP2 genes (48). miRNA-122 affects CAD by regulating genes associated with lipid synthesis and oxidation, including SREBP, microsomal triglyceride transfer protein, Krüppel-like factor 6 and cationic amino acid transporter 1 (49). Silencing of miRNA-122 leads to a decrease in total cholesterol and triglyceride in plasma (49). At present, lncRNAs have emerged as critical factors in the promotion of hepatic gluconeogenesis and cholesterol biosynthesis via the SREBP pathway (46). However, their functions in CAD remain unknown, which highlights the importance of the results of the present study.

In conclusion, a novel lncRNA ENST00000416361 was first identified to be significantly upregulated in patients with CAD by high-throughput sequencing in the present study. lncRNA ENST00000416361 has potential as a biomarker for clinical screening and diagnosis of CAD. The experimental results indicated that ENST00000416361 had an association with 2 inflammatory cytokines (IL- 6 and TNF- $\alpha$ ) and the lipid metabolism-associated genes (SREBP1 and SREBP2). These results suggested that ENST00000416361 may be related to the pathogenesis of atherosclerosis, such as inflammation and lipid metabolism. Nevertheless, CAD is often accompanied by hypertension, and its occurrence and development are also affected by numerous environmental factors. Although these parameters are markedly associated with CAD, it is difficult to independently evaluate the effect of each factor, therefore this should be investigated in future studies. Therefore, the specific mechanism underlying ENST00000416361 in the progression of $\mathrm{CAD}$ has not been elucidated, which requires further exploration.

\section{Acknowledgements}

Not applicable.

\section{Funding}

This work was financially supported by the Special Diagnosis Techniques for Clinical Key Diseases of Suzhou Municipal Health and Family Planning Commission (grant no.LCZX201610),Scienceand Technology DevelopmentFund of Nanjing Medical University (grant no. NMUB2018219) and the Suzhou Basic Research in Medical and Health Application Grant (grant no. sys2018090).

\section{Availability of data and materials}

The datasets used and/or analyzed during the current study are available from the corresponding author on reasonable request.

\section{Authors' contributions}

KS designed and funded this study. PL was the principal experimenter and drafted the manuscript. XY assisted the experiment and provided statistical analysis. QC designed the study and revising this manuscript critically for important intellectual content. GX, JW, and ZP performed the collection and interpretation of data. JY, ML and LY conducted literature search and data interpretation. All authors reviewed the results and approved the final version of the manuscript.

\section{Ethics approval and consent to participate}

The study was approved by the Ethics Committee of Nanjing Medical University. Informed consent was obtained from the patients and their families.

\section{Patient consent for publication}

Not applicable.

\section{Competing interests}

The authors declare that they have no competing interests.

\section{References}

1. Pagliaro BR, Cannata F, Stefanini GG and Bolognese L: Myocardial ischemia and coronary disease in heart failure. Heart Fail Rev 25: 53-65, 2020

2. Sayols-Baixeras S, Luis-Ganella C, Lucas G and Elosua R: Pathogenesis of coronary artery disease: focus on genetic risk factors and identification of genetic variants. Appl Clin Genet 7: 15-32, 2014.

3. Glass CK and Witztum JL: Atherosclerosis. The road ahead. Cell 104: 503-516, 2001

4. Dalen JE, Alpert JS, Goldberg RJ and Weinstein RS: The epidemic of the 20(th) century: Coronary heart disease. The American journal of medicine 127: 807-812, 2014.

5. Dai X, Wiernek S, Evans JP and Runge MS: Genetics of coronary artery disease and myocardial infarction. World J Cardiol 8: 1-23, 2016.

6. Meseure D, Drak Alsibai K, Nicolas A, Bieche I and Morillon A: Long noncoding RNAs as new architects in cancer epigenetics, prognostic biomarkers, and potential therapeutic targets. Biomed Res Int 2015: 320214, 2015.

7. Esteller M: Non-coding RNAs in human disease. Nat Rev Genet 12: 861-874, 2011.

8. Hueso M, Cruzado JM, Torras J and Navarro E: ALUminating the path of atherosclerosis progression: Chaos theory suggests a role for Alu repeats in the development of atherosclerotic vascular disease. Int J Mol Sci 19: E1734, 2018.

9. Yang F, Xue X, Bi J, Zheng L, Zhi K, Gu Y and Fang G: Long noncoding RNA CCAT1, which could be activated by c-Myc, promotes the progression of gastric carcinoma. J Cancer Res Clin Oncol 139: 437-445, 2013.

10. Yuan JH, Yang F, Wang F, Ma JZ, Guo YJ, Tao QF, Liu F, Pan W, Wang TT, Zhou CC, et al: A long noncoding RNA activated by TGF-beta promotes the invasion-metastasis cascade in hepatocellular carcinoma. Cancer Cell 25: 666-681, 2014.

11. Leung A and Natarajan R: Noncoding RNAs in vascular disease. Curr Opin Cardiol 29: 199-206, 2014.

12. Bitarafan S, Yari M, Broumand MA, Ghaderian SM, Rahimi M, Mirfakhraie R, Azizi F and Omrani MD: Association of increased levels of lncRNA H19 in PBMCs with risk of coronary artery disease. Cell J 20: 564-568, 2019.

13. Zhang Z, Gao W, Long QQ, Zhang J, Li YF, Liu DC, Yan JJ, Yang ZJ and Wang LS: Increased plasma levels of lncRNA H19 and LIPCAR are associated with increased risk of coronary artery disease in a Chinese population. Sci Rep 7: 7491, 2017.

14. Yu D, Tang C, Liu P, Qian W and Sheng L: Withdrawal notice: Targeting lncRNAs for cardiovascular therapeutics in coronary artery disease. Curr Pharm Des: Jan 8, 2018 (Epub ahead of print). doi: 10.2174/1381612824666180108120727.

15. Wu G, Cai J, Han Y, Chen J, Huang ZP, Chen C, Cai Y, Huang $\mathrm{H}$, Yang Y, Liu Y, et al: LincRNA-p21 regulates neointima formation, vascular smooth muscle cell proliferation, apoptosis, and atherosclerosis by enhancing p53 activity. Circulation 130: 1452-1465, 2014. 
16. Tang SS, Cheng J, Cai MY, Yang XL, Liu XG, Zheng BY and Xiong XD: Association of lincRNA-p21 haplotype with coronary artery disease in a Chinese Han population. Dis Markers 2016: 9109743, 2016.

17. Cai Y, Yang Y, Chen X, Wu G, Zhang X, Liu Y, Yu J, Wang X, Fu J, Li C, et al: Circulating 'IncRNA OTTHUMT00000387022' from monocytes as a novel biomarker for coronary artery disease. Cardiovasc Res 112: 714-724, 2016.

18. Yang Y, Cai Y, Wu G, Chen X, Liu Y, Wang X, Yu J, Li C, Chen X, Jose PA, et al: Plasma long non-coding RNA, CoroMarker, a novel biomarker for diagnosis of coronary artery disease. Clin Sci (Lond) 129: 675-685, 2015.

19. Wenger NK: 2011 ACCF/AHA focused update of the guidelines for the management of patients with Unstable Angina/Non-ST-Elevation Myocardial Infarction (updating the 2007 Guideline): highlights for the clinician. Clin Cardiol 35: 3-8, 2012.

20. Jaatinen $\mathrm{T}$ and Laine $\mathrm{J}$ : Isolation of mononuclear cells from human cord blood by Ficoll-Paque density gradient. Curr Protoc Stem Cell Biol: Jun 1, 2007 (Epub ahead of print). doi: org/10.1002/9780470151808.sc02a01s1.

21. Jia Y, Xu H, Li Y, Wei C, Guo R, Wang F, Wu Y, Liu J, Jia J, Yan J, et al: A Modified Ficoll-Paque gradient method for isolating mononuclear cells from the peripheral and umbilical cord blood of humans for biobanks and clinical laboratories. Biopreserv Biobank 16: 82-91, 2018.

22. Trapnell C, Hendrickson DG, Sauvageau M, Goff L, Rinn JL and Pachter L: Differential analysis of gene regulation at transcript resolution with RNA-seq. Nat Biotechnol 31: 46-53, 2013.

23. Ashburner M, Ball CA, Blake JA, Botstein D, Butler $\mathrm{H}$, Cherry JM, Davis AP, Dolinski K, Dwight SS, Eppig JT, et al: Gene ontology: Tool for the unification of biology. The Gene Ontology Consortium. Nat Genet 25: 25-29, 2000.

24. The Gene Ontology Consortium: The Gene Ontology Resource: 20 years and still GOing strong. Nucleic Acids Res 47: D330-D338, 2019.

25. Huang M,Zhong Z,Lv M,Shu J,Tian Q and Chen J: Comprehensive analysis of differentially expressed profiles of lncRNAs and circRNAs with associated co-expression and ceRNA networks in bladder carcinoma. Oncotarget 7: 47186-47200, 2016.

26. Agarwal V, Bell GW, Nam JW and Bartel DP: Predicting effective microRNA target sites in mammalian mRNAs. Elife 4: e05005, 2015.

27. Garcia DM, Baek D, Shin C, Bell GW, Grimson A and Bartel DP: Weak seed-pairing stability and high target-site abundance decrease the proficiency of 1 sy- 6 and other microRNAs. Nat Struct Mol Biol 18: 1139-1146, 2011.

28. Friedman RC, Farh KK, Burge CB and Bartel DP: Most mammalian mRNAs are conserved targets of microRNAs. Genome Res 19: 92-105, 2009.

29. Grimson A, Farh KK-H, Johnston WK, Garrett-Engele P, Lim LP and Bartel DP: MicroRNA targeting specificity in mammals: determinants beyond seed pairing. Mol Cell 27: 91-105, 2007.

30. Lewis BP, Burge CB and Bartel DP: Conserved seed pairing, often flanked by adenosines, indicates that thousands of human genes are microRNA targets. Cell 120: 15-20, 2005.

31. John B, Enright AJ, Aravin A, Tuschl T, Sander C and Marks DS: Human MicroRNA targets. PLoS Biol 2: e363-e363, 2004.

32. Shannon P, Markiel A, Ozier O, Baliga NS, Wang JT, Ramage D, Amin N, Schwikowski B and Ideker T: Cytoscape: A software environment for integrated models of biomolecular interaction networks. Genome Res 13: 2498-2504, 2003.

33. Iliopoulos D, Drosatos K, Hiyama Y, Goldberg IJ and Zannis VI: MicroRNA-370 controls the expression of microRNA-122 and Cpt1alpha and affects lipid metabolism. J Lipid Res 51: $1513-1523,2010$

34. Livak KJ and Schmittgen TD: Analysis of relative gene expression data using real-time quantitative PCR and the 2(-Delta Delta C(T)) method. Methods 25: 402-408, 2001.
35. Hou S, Fang M, Zhu Q, Liu Y, Liu L and Li X: MicroRNA-939 governs vascular integrity and angiogenesis through targeting gamma-catenin in endothelial cells. Biochem Biophys Res Commun 484: 27-33, 2017.

36. Jaé N, Heumüller AW, Fouani Y and Dimmeler S: Long non-coding RNAs in vascular biology and disease. Vascul Pharmacol 114: 13-22, 2019.

37. Ahmadi R, Heidarian E, Fadaei R, Moradi N, Malek M and Fallah S: miR-342-5p Expression levels in coronary artery disease patients and its association with inflammatory cytokines. Clin Lab 64: 603-609, 2018.

38. Fadaei R, Moradi N, Kazemi T, Chamani E, Azdaki N, Moezibady SA, Shahmohamadnejad S, Fallah S, et al: Decreased serum levels of CTRP12/adipolin in patients with coronary artery disease in relation to inflammatory cytokines and insulin resistance. Cytokine 113: 326-331, 2019.

39. Libby P, Tabas I, Fredman G and Fisher EA: Inflammation and its resolution as determinants of acute coronary syndromes. Circ Res 114: 1867-1879, 2014.

40. Hammadah M, Sullivan S, Pearce B, Mheid IA, Wilmot K, Ramadan R, Tahhan AS, O'Neal WT, Obideen M, Alkhoder A, et al: Inflammatory response to mental stress and mental stress induced myocardial ischemia. Brain Behav Immun 68: 90-97, 2018.

41. Chen H, Ding S, Liu X, Wu Y and Wu X: Association of interleukin- 6 genetic polymorphisms and environment factors interactions with coronary artery disease in a Chinese Han population. Clin Exp Hypertens 40: 514-517, 2018.

42. Guo F, Tang C, Li Y, Liu Y, Lv P, Wang W and Mu Y: The interplay of LncRNA ANRIL and miR-181b on the inflammation-relevant coronary artery disease through mediating NF-kappaB signalling pathway. J Cell Mol Med 22: 5062-5075, 2018.

43. Kuan YC, Hashidume T, Shibata T, Uchida K, Shimizu M, Inoue $\mathrm{J}$ and Sato R: Heat shock protein 90 modulates lipid homeostasis by regulating the stability and function of sterol regulatory element-binding protein (SREBP) and SREBP cleavage-activating protein. J Biol Chem 292: 3016-3028, 2017.

44. Brown MS and Goldstein JL: The SREBP pathway: regulation of cholesterol metabolism by proteolysis of a membrane-bound transcription factor. Cell 89: 331-340, 1997.

45. Sato R: Sterol metabolism and SREBP activation. Arch Biochem Biophys 501: 177-181, 2010.

46. Perez-Belmonte LM, Moreno-Santos I, Cabrera-Bueno F, Sánchez-Espín G, Castellano D, Such M, Crespo-Leiro MG, Carrasco-Chinchilla F, Alonso-Pulpón L,López-Garrido M, et al: Expression of Sterol Regulatory Element-Binding Proteins in epicardial adipose tissue in patients with coronary artery disease and diabetes mellitus: Preliminary study. Int J Med Sci 14: 268-274, 2017.

47. Abudesimu A, Adi D, Siti D, Xie X, Yang YN, Li XM, Wang YH, Wang YT, Meng YJ, Liu F, et al: Association of genetic variations in the lipid regulatory pathway genes FBXW7 and SREBPs with coronary artery disease among Han Chinese and Uygur Chinese populations in Xinjiang, China. Oncotarget 8: 88199-88210, 2017.

48. Price NL, Singh AK, Rotllan N, Goedeke L, Wing A, Canfrán-Duque A, Diaz-Ruiz A, Araldi E, Baldán A, Camporez JP, et al: Genetic ablation of miR-33 increases food intake, enhances adipose tissue expansion, and promotes obesity and insulin resistance. Cell Rep 22: 2133-2145, 2018.

49. Dong J, Liang YZ, Zhang J, Wu LJ, Wang S, Hua Q and Yan YX: Potential role of lipometabolism-related MicroRNAs in peripheral blood mononuclear cells as biomarkers for coronary artery disease. J Atheroscler Thromb 24: 430-441, 2017.

This work is licensed under a Creative Commons Attribution-NonCommercial-NoDerivatives 4.0 International (CC BY-NC-ND 4.0) License. 